\title{
Practices for Effective Software Project Management in Global Software Development: A Systematic Literature Review
}

\author{
Muhammad Yaseen \\ City University of Science and \\ Information Technology \\ Peshawar, Pakistan
}

\author{
Said Nabi \\ Department of Computer Science \\ COMSATS University Islamabad \\ Islamabad
}

\author{
Muhammad Ali Awan \\ City University of Science and \\ Information Technology \\ Peshawar, Pakistan \\ Sadiq Khan \\ Department of Computer Science, \\ International Islamic University \\ Islamabad
}

\author{
Atta Ur Rahman \\ Department of Computer Science \\ COMSATS University Islamabad \\ Islamabad
}

\author{
Mohim Bacha \\ Department of Computer Software \\ Engineering UET
}

Peshawar

\begin{abstract}
Effective software project management plays an important role during requirements collection and implementation for any software system. In Global Software Development (GSD), its significance increase more as stakeholders are far away across the globe. In GSD, challenges such as language differences and time zone differences cause significant barrier during requirements collection and thus need of effective project management increase more and more to handle challenges of GSD. This study address possible solutions and practices for effective global software project management. Through Systematic Literature Review (SLR), 25 practices are identified. These practices will help software vendors to better manage software projects in GSD.
\end{abstract}

\section{Keywords}

Practices, Effective software project management, Global Software Development, Systematic Literature Review.

\section{INTRODUCTION}

Requirement Engineering (RE) deals with all aspects of software requirements from requirements collection to requirements implementation in systematic way [1][2]. RE consist of different phases. In requirement elicitation phase, requirements for software system are collected from clients by applying various elicitation techniques such as background study, interview, questionnaire, apprenticing [3][4]. In GSD, where clients and vendors are far away and there exist geographical distance thus proper requirements collection and management become more difficult. According to [5], due to barriers such as geographical distance, time zone differences and language differences, effective requirements collection and implementation in GSD become more and more difficult which can affect the quality of software systems and project completion time and cost [6]. In GSD, where there exist critical challenges during requirements elicitation, proper requirements management and implementation become a big challenge. In GSD, there is essential to implement practices needed for successful management of software project. The purpose of this research is to identify all possible practices for effective global project management. No SLR has been yet conducted to identify best practices for global project management. This current work will fill this research gap. Implementing all possible best practices will help software vendors to better implement RE in GSD.

\section{BACKGROUND STUDY}

Systematic literature review is conducted by [7], where effective communication is identified as most critical success factor during successful requirements implementation in GSD. With effective communication channels, proper elicitation of requirements in GSD can be assured.

Language is very essential in requirement collection as it disturbs transfer of knowledge and proper communication that depends entirely on appropriate usage of language [8]. In GSD this factor is a big challenge because mostly the clients and vendors face difficulties to fully cope the terminologies of other languages [9][10]. In GSD, timely communication is another big challenge because there exist difference of time zones among countries and sometimes this variation in time zones can increases that makes it difficult to communicate synchronously such as video or audio calls etc [7]. Time zone variance is a challenge for both vendors and clients in GSD and thus it requires appropriate solutions and practices [11][12].

Elicitation problems occur due to lack of proper communication and for which model is suggested in one of the studies [13]. The first phase in this model is to arrange interviews with several software industries and compare the consequences with outcomes as identified by research group. Theoretical modeling of requirements uncertainty and elicitation dimensions is the next step and the last phase of the model is the validation of model from different software organizations.

Communicating knowledge and information's in GSD is challenging and a big challenge [14]. Poor SRS shows that the knowledge managing was not properly conducted [15].

Proper discussion and negotiation on requirements in GSD is an crucial challenge to be overcome and but due to the stated challenges such as time differences, language barriers and culture differences it is quite difficult to have proper negotiations on requirements [16][17]. Trust should be established in GSD because without trust no team is possible and without team no collaboration is possible and without collaboration and trust a success is achieved only by luck [17].

Different implementation models are suggested for efficient requirements implementation in the context of GSD. 
Requirement Implementation Model (RIM)[18], Requirement Elicitation Model (REM) [19] and Requirement Management Model (RMM)[20] are presented in different studies. The proposed models are based on empirical studies that will consist of all possible challenges and success factors during requirements implementation, elicitation and management in context of GSD.

\section{RESEARCH METHOD}

SLR is conducted to achieve goals and objectives of the research. In our previous studies we used the same research method to achieve other goals [21][22]. In this research study, we are repeating the same steps as we did in our previous research works. The step by step approach of conducting SLR is shown in Fig 1.

\section{a. Research Question Identification}

The first step of doing SLR is finalizing research questions. Based on particular research question, data are retrieved from different research studies. The following research question (RQ1) for this study.

RQ1: What are possible solutions and practices for implementation of effective project management in global software development?

\section{b. Literature Search Strategy}

Literature Search strategy is made that consist of the following;
- $\quad$ Range of search ( time and space)

There is no bound on search time and space. Publications related to our defined RQ1 will be included for the next step.

- $\quad$ Electronic data sources used

We will used Science Direct, Springer link, ACM portal, IEEE Xplore.

\section{- Strings for the search}

The following search string is finalized for this research work. (("Requirement Engineering" OR "Requirement Implementation" OR "Requirement Elicitation") AND ("Global Software development" OR "Distributed Software Development”)).

\section{c. Literature Selection Criteria}

During literature selection criteria, not all but some publications that are most relevant to research question are selected for final data retrieval. Studies that are not written in English language will be excluded from final list of papers. Inclusion and exclusion criteria for this research study is based on RQ1. Papers that address practices for effective communication in GSD will be only included.

\section{d. Data extraction}

This is last phase where practices for effective communication during requirements implementation in context of GSD will be retrieved from final selected list of publications.

Based on inclusion and exclusion criteria, Table 1 shows number of primary and final selected papers from different digital resources.

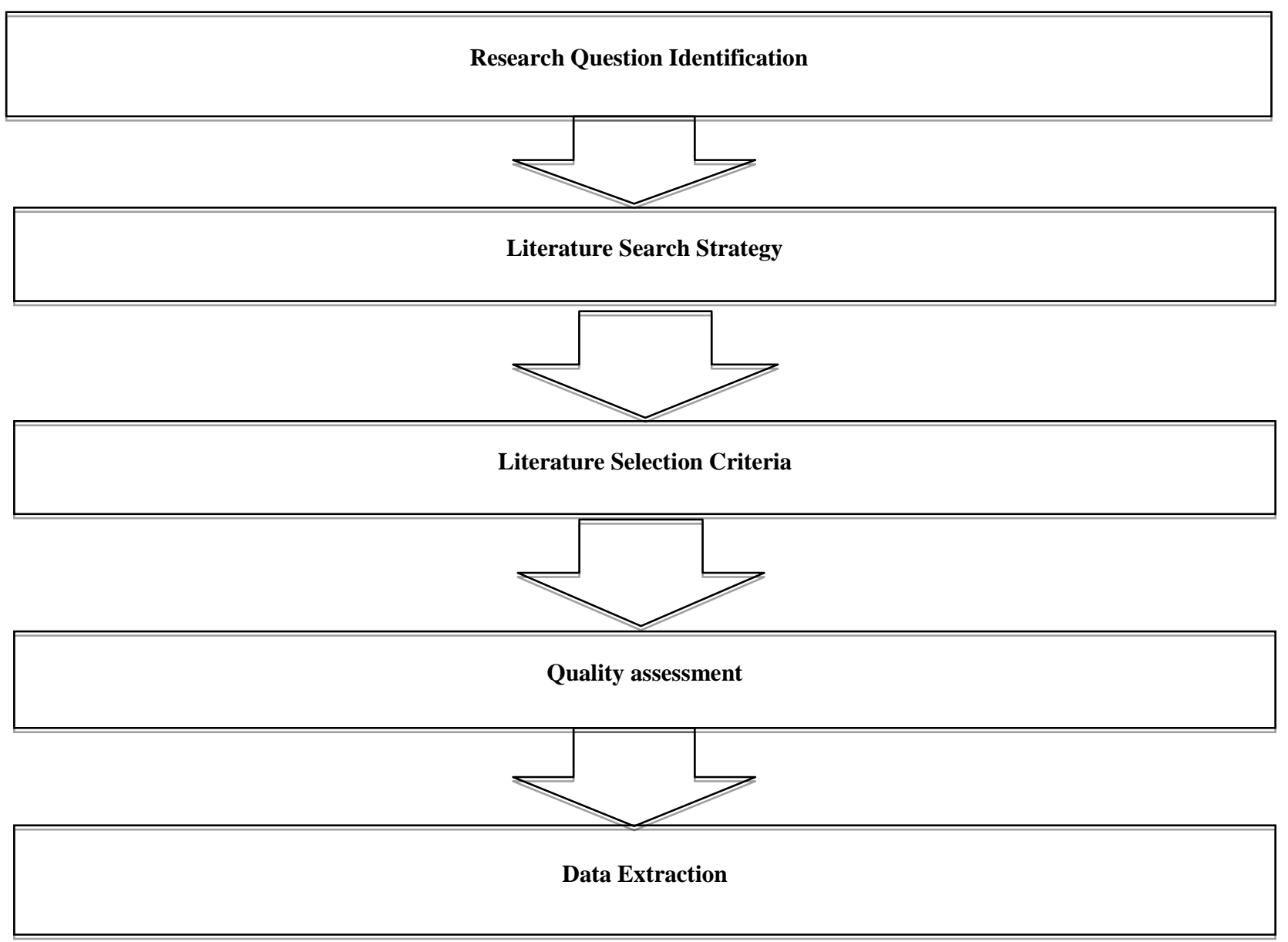

Fig 1: Development Process for the SLR Protocol 
Table 1: Number of publications selected for data extraction

\begin{tabular}{|c|c|c|c|}
\hline Publisher Site & Total Results found & Primary selection & $\begin{array}{c}\text { Final Selected Papers } \\
\text { (Appendix) }\end{array}$ \\
\hline IEEExplore & 360 & 85 & 14 \\
\hline Science Direct & 300 & 85 & 3 \\
\hline ACM & 280 & 40 & 7 \\
\hline Others & 430 & 20 & 3 \\
\hline SpringerLink & 140 & 335 & 30 \\
\hline TOTAL & 1510 & & 4 \\
\hline
\end{tabular}

\section{REULTS AND DISCUSSION}

After conducting SLR, following 25 practices for implementation of effective software project management

Table 2: Practices for effective project management in GSD

\begin{tabular}{|c|c|c|}
\hline S/No & Practices for effective project management in GSD & $\begin{array}{l}\text { No of Practices } \\
\text { via } S L R(N=30)\end{array}$ \\
\hline 1 & Global project management tools should be used & 5 \\
\hline 2 & Clear definition of roles and responsibilities and make team wisely & 6 \\
\hline 3 & Strong leadership and skills at the project management & 4 \\
\hline 4 & Bring collaboration in the team & 2 \\
\hline 5 & Professionally train all project managers & 2 \\
\hline 6 & Apply best practices from the CMMI & 2 \\
\hline 7 & Information about best elicitation techniques used in market & 1 \\
\hline 8 & Obtaining information about the organizational environment & 1 \\
\hline 9 & Knowledge of requirements documentation basics & 1 \\
\hline 10 & Knowledge on definition of requirements is must for the team & 1 \\
\hline 11 & Management support for people in GSD is necessary & 1 \\
\hline 12 & Regular personal meetings & 1 \\
\hline 13 & Work with multiple partners. Distribute critical knowledge & 1 \\
\hline 14 & Have periodic workshops with teams. & 1 \\
\hline 15 & Formalization of documentation & 1 \\
\hline 16 & Develop a trust relationship in the team & 3 \\
\hline 17 & peer-to-peer links at all management & 1 \\
\hline 18 & Innovative processes and technologies & 5 \\
\hline 19 & Use agile practices & 3 \\
\hline 20 & Creating a communication coordinator role to reduce misinterpretations & 1 \\
\hline 21 & Use a human facilitator in integrated rich communication media during decision making & 1 \\
\hline 22 & Implement and systematically follow quality gates at work product level & 2 \\
\hline 23 & Maintain an organization risk repository. & 1 \\
\hline 24 & organizations need to allow enough time to make the change & 1 \\
\hline 25 & Rigorously highlight insufficient quality & 1 \\
\hline
\end{tabular}

during requirements implementation in GSD as shown in Table 2 are identified. 
Modern global project management tools should be used for effective implementation of software requirements. Modern GSD tools facilitate both synchronous and asynchronous ways of communication among clients and vendors. In GSD, clear roles and responsibilities should be assigned to each member of the team. As compare to local development, in GSD, role of strong and effective leadership become more important. Strong leadership can enable team members to effectively manage requirements. Bring collaboration and coordination in team makes it easier to share knowledge in GSD which will assist successful requirements implementation. Professional training of team members for GSD is a success factor for global project management. Vendors of GSD need to apply best practices from CMMI. As in GSD, traditional ways of elicitation techniques such as background study is not possible so vendors need to implement collaborative ways of elicitation techniques and for this purpose they need to know from surrounding organizations about use of new ways of elicitation techniques in GSD. Knowledge on requirements and its way of implementation is necessary to understand before implementation. Knowledge comes with experience and for this purpose, it is necessary to access experience persons inside or outside organizations. Support of management to bring best practices in GSD is very important. Meetings of members on regular basis is very important in GSD. Through regular meetings, any knowledge and information's that can favor successful requirements implementation can be shared among team members. It is the responsibility of project managers to arrange workshops on regular basis for their team members on how to handle software projects in GSD. Through workshops and discussion, any conflicts on requirements can be resolved efficiently. Formalization of documents is important in GSD which makes it easier to track any information on requirements from clients. Trust building is considered to be very important success factor among team members and with clients of GSD. It is the responsibility of project managers to bring trust with clients. Peer to peer links among team members bring more collaboration and coordination and thus favor knowledge sharing and helps in conflict resolution among team members in GSD. Use of innovative technologies such as semantic web technology and use of ontologies for requirements representation and management in GSD are suggested by many authors for effective requirements collection and management. Use of modern software process models such as agile helps to better implement requirements in GSD and for this purpose more and more work is already done. As in GSD, their exist difference in languages and terminologies among vendors and clients, so defining role of communication coordinator that facilitate effective communication in GSD is very helpful and thus proper negotiations on requirements become easy. As culture problem is also considered as critical challenge in GSD, thus defining coordinator role makes it easier to reduce culture gap among stakeholders. Similarly during meetings of management, human facilitator role for effective rich communication become more helpful. Making risk repository in GSD that contain possible risks that can occur in GSD and factors that are important to tackle these risks should be defined so that possibility of any potential risks can be resolved on time. Requirements change management is GSD is more difficult as compare to local development, thus proper time should be given before doing some changes in requirements. Fig 2 shows critical practices that have more frequency of occurrence from literature. Other practices that have low frequency are also important.

\section{Critical Practices}

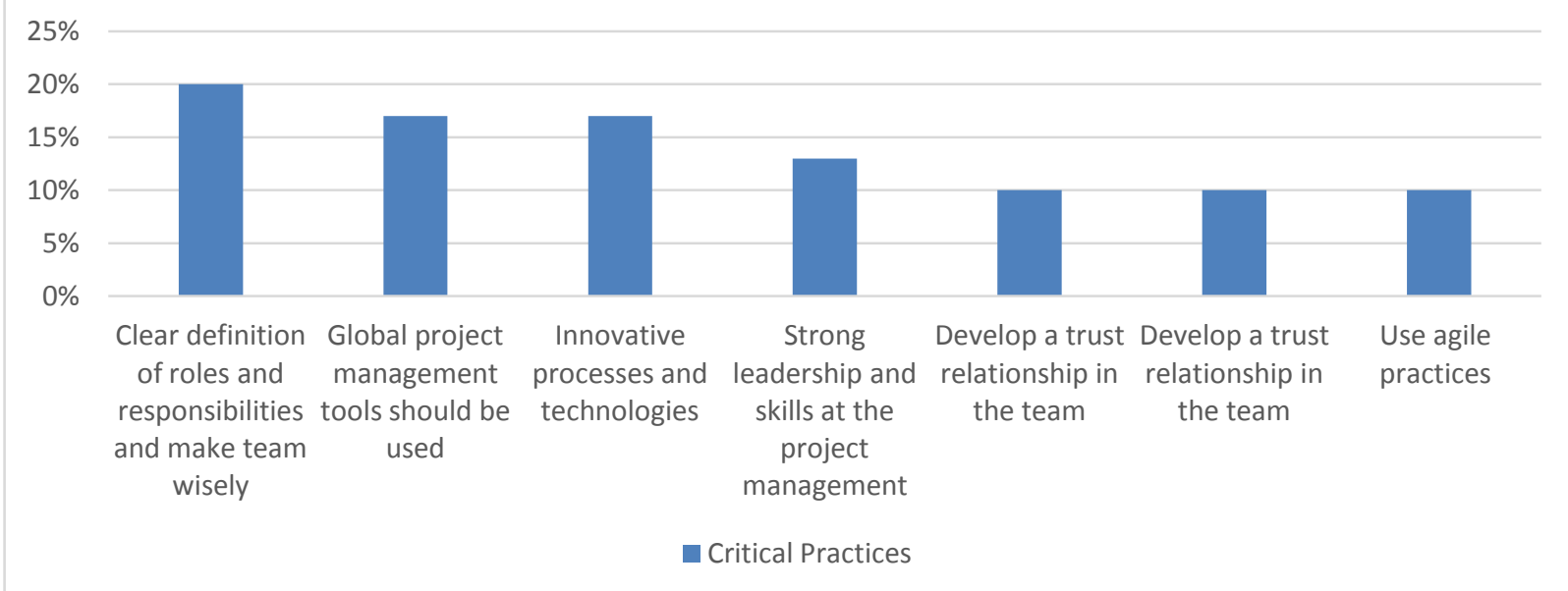

Fig 2: Critical Practices identified with SLR

\section{CONCLUSION AND FUTURE WORK}

As a result of SLR, 25 practices are identified for implementation of effective software project management in GSD. In these practices, Global project management tools should be used, innovative processes and technologies, clear definition of roles and responsibilities and make team wisely, strong leadership and skills at the project management, develop a trust relationship in the team and use agile practices are maximum identified practices from literature. Implementing these practices will assist software vendors of GSD to efficiently manage software projects. Effective management practices will result in delivery of quality product which is very difficult in GSD. In future, the aim is to validate these practices through industrial surveys and explore some new practices that are not previously identified from literature.

\section{REFERENCES}

[1] M. Yaseen, A. Mustapha, and N. Ibrahim, 'MINIMIZING INTER-DEPENDENCY ISSUES OF REQUIREMENTS IN PARALLEL DEVELOPING SOFTWARE PROJECTS WITH AHP', vol. 8, no. Viii, 2019. 
[2] M. Yaseen, A. Mustapha, and N. Ibrahim, 'Prioritization of Software Functional Requirements: Spanning Tree based Approach', vol. 10, no. 7, pp. 489-497, 2019.

[3] M. Yaseen, A. Mustapha, and N. Ibrahim, 'An Approach for Managing Large-Sized Software Requirements During Prioritization', 2018 IEEE Conf. Open Syst., pp. 98-103, 2019.

[4] M. Yaseen, N. Ibrahim, and A. Mustapha, 'Requirements Prioritization and using Iteration Model for Successful Implementation of Requirements', Int. J. Adv. Comput. Sci. Appl., vol. 10, no. 1, pp. 121-127, 2019.

[5] M. Yaseen, S. Baseer, and S. Sherin, 'Critical Challenges for Requirement Implementation in Context of Global Software Development: A Systematic Literature Review', pp. 120-125, 2015.

[6] Z. Ali, M. Yaseen, and S. Ahmed, 'Effective communication as critical success factor during requirement elicitation in global software development', vol. 8, no. 03, pp. 108-115, 2019.

[7] M. Yaseen and Z. Ali, 'Success Factors during Requirements Implementation in Global Software Development: A Systematic Literature Review', vol. 8, no. 3, pp. 56-68, 2019.

[8] T. Illes-Seifert, A. Herrmann, M. Geisser, and T. Hildenbrand, 'The Challenges of Distributed Software Engineering and Requirements Engineering: Results of an Online Survey', 1st Int. Glob. Requir. Eng. Work. GREW 2007, pp. 55-65, 2007.

[9] M. Yaseen and Z. Ali, 'Practices for Effective Communication during Requirements Elicitation in Global Software Development', vol. 8, no. 06, pp. 240245, 2019.

[10] A. U. Rahman, M. Yaseen, and Z. Ali, 'Identification of Practices for Proper Implementation of Requirements in Global Software Development: A Systematic Literature Review Protocol', vol. 177, no. 13, pp. 53-58, 2019.

[11] D. Damian, 'An empirical study of requirements engineering in distributed software projects : is distance negotiation more effective ?', vol. 4, no. 04, pp. 149-152, 2001

[12] I. Kwan, D. Damian, and S. Marczak, 'The effects of distance, experience, and communication structure on requirements awareness in two distributed industrial software projects', Glob. Requir. Eng. Work. conj. Intl Conf Glob. Softw. Eng. 2007., no. May, 2007.

[13] W. Hussain, J. Buchan, and T. Clear, 'Managing Requirements in Globally Distributed COTS Customization', Proc. - Int. Comput. Softw. Appl. Conf., vol. 18-21-Augu, pp. 33-38, 2014.

[14] D. E. Damian and D. Zowghi, 'RE challenges in multisite software development organisations', no. 2003, pp. 149-160, 2007.

[15] M. Yaseen, Z. Ali, and A. U. R. Rahman, 'RESEARCH PAPERS ROLE OF SOFTWARE DEVELOPER' $S$ HAPPINESS IN PROJECTS SUCCESS : A PROPOSED DEVELOPERS HAPPINESS MODEL ( DHM ) By', vol. 14, no. 1, 2019.
[16] R. Lai and N. Ali, 'A Requirements Management Method for Global Software Development', Humanpub.Org, vol. 1, no. March, pp. 38-58, 2013.

[17] A. M. Søderberg, S. Krishna, and P. Bjørn, 'Global software development: Commitment, trust and cultural sensitivity in strategic partnerships', J. Int. Manag., vol. 19, no. 4, pp. 347-361, 2013.

[18] M. Yaseen, S. Baseer, S. Ali, S. U. Khan, and Abdullah, 'Requirement implementation model (RIM) in the context of global software development', 2015 Int. Conf. Inf. Commun. Technol. ICICT 2015, 2016.

[19] M. Yaseen and U. Farooq, 'Requirement Elicitation Model (REM) in the Context of Global Software Development', Glob. J. Comput. Sci. Technol., vol. 1, no. 2, pp. 1-6, 2018.

[20] M. Yaseen, Z. Ali, and M. Humayoun, 'Requirements Management Model (RMM): A Proposed Model for Successful Delivery of Software Projects', Int. J Comput. Appl., vol. 178, no. 17, pp. 32-36, 2019.

[21] M. Yaseen, S. Ali, . A., and N. Ullah, 'An Improved Framework for Requirement Implementation in the context of Global Software Development: A Systematic Literature Review Protocol', Int. J. Database Theory Appl., vol. 9, no. 6, pp. 161-170, 2016.

[22] Z. Ali and M. Yaseen, 'Critical Challenges for Requirement Implementation in Global Software Development: A Systematic Literature Review Protocol with Preliminary Results', vol. 182, no. 48, pp. 17-23, 2019.

\section{APPENDIX [List of papers]}

[s1] M. Heindl and S. Biffl, 'Risk management with enhanced tracing of requirements rationale in highly distributed projects', Proc. 2006 Int. Work. Glob. Softw. Dev. Pract. - GSD ’06, p. 20, 2006.

[s2] B. Berenbach, 'Impact of Organizational Structure on Distributed Requirements Engineering Processes: Lessons Learned', Int. Work. Glob. Softw. Dev. Pract. GSD 06, pp. 15-19, 2006.

[s3] C. Gutwin, R. Penner, and K. Schneider, 'Group Awareness in Distributed Software Development', pp. 72-81, 2004.

[s4] C. L. Campbell, B. V. A. N. D. E. Walle, and A. G. S. Development, 'Asynchronous Requirements Engineering: Enhancing Distributed Software Development', pp. 133-136, 2003.

[s5] D. Damlan, 'Stakeholders in global requirements engineering: Lessons learned from practice', IEEE Softw., vol. 24, no. 2, pp. 21-27, 2007.

[s6] D. Damian, 'An empirical study of requirements engineering in distributed software projects : is distance negotiation more effective ?', vol. 4, no. 04, pp. 149-152, 2001.

[s7] D. Requirements, 'Enabling Collaboration in Distributed Requirements Management - ABI INFORM Collection ProQuest', 2006

[s8] a. Lopez, J. Nicolas, a. Toval, J. M. Carrillo-de-Gea, and a. Toval, 'Risks and Safeguards for the Requirements Engineering Process in Global Software 
Development', Glob. Softw. Eng. 2009. ICGSE 2009. Fourth IEEE Int. Conf., no. 1, pp. 394-399, 2009.

[s9] M. Geisser, T. Hildenbrand, F. Rothlauf, and C. Atkinson, 'An Evaluation Method for Requirements Engineering Approaches in Distributed Software Development Projects', no. Icsea, 2007.

[s10] M. Romero, A. Vizcaino, and M. Piattini, 'Teaching Requirements Elicitation within the Context of Global Software Development', Comput. Sci. (ENC), 2009 Mex. Int. Conf., pp. 232-239, 2009.

[s11] J. Iqbal, R. Ahmad, M. H. Nizam, M. Nasir, and M. A. Noor, 'Significant Requirements Engineering Practices for Software Development Outsourcing', Proc. 2013 \{22Nd\} Aust. Conf. Softw. Eng., pp. 137-144, 2013 .

[s12] W. Hussain and T. Clear, 'Spreadsheets as Collaborative Technologies in Global Requirements Change Management', 2014.

[s13] C. Ebert, B. K. Murthy, and N. N. Jha, 'Managing Risks in Global Software Engineering: Principles and Practices', 2008 IEEE Int. Conf. Glob. Softw. Eng., pp. 131-140, 2008.

[s14] Z. Haag, R. Foley, and J. Newman, 'Software process improvement in geographically distributed software engineering: an initial evaluation', EUROMICRO 97. Proc. 23rd EUROMICRO Conf. New Front. Inf. Technol. (Cat. No.97TB100167), pp. 134$141,1997$.

[s15] M. Korkala and P. Abrahamsson, 'Communication in Distributed Agile Development: A Case Study', 33rd EUROMICRO Conf. Softw. Eng. Adv. Appl. (EUROMICRO 2007), no. Seaa, pp. 203-210, 2007.

[s16] J. M. M. Bhat, M. Gupta, and S. N. N. Murthy, 'Overcoming Requirements Engineering Challenges: Lessons from Offshore Outsourcing', IEEE Softw., vol. 23, no. 5, pp. 38-44, 2006.

[s17] A. Boden, B. Nett, and V. Wulf, 'Coordination practices in distributed software development of small enterprises', Proc. - Int. Conf. Glob. Softw. Eng. ICGSE 2007, no. Icgse, pp. 235-246, 2007.

[s18] M. Kauppinen, M. Vartiainen, J. Kontio, S. Kujala, and R. Sulonen, 'Implementing requirements engineering processes throughout organizations: Success factors and challenges', Inf. Softw. Technol., vol. 46, no. 14, pp. 937-953, 2004. [s19] I. Inayat, S. Salwah, S. Marczak, M. Daneva, and S. Shamshirband, 'Computers in Human Behavior A systematic literature review on agile requirements engineering practices and challenges', Comput. Human Behav., 2014

[s20] M. Daneva et al., 'The Journal of Systems and Software Agile requirements prioritization in large-scale outsourced system projects : An empirical study', J. Syst. Softw., vol. 86, no. 5, pp. 1333-1353, 2013.

[s21] D. M. Fernández and S. Wagner, 'Naming the pain in requirements engineering: A design for a global family of surveys and first results from Germany', Inf. Softw. Technol., vol. 57, no. 1, pp. 616-643, 2015.

[s22] G. N. Aranda, A. Vizcaíno, and M. Piattini, 'A framework to improve communication during the requirements elicitation process in GSD projects', Requir. Eng., vol. 15, no. 4, pp. 397-417, 2010.

[s23] A. Lamersdorf and J. Münch, 'A multi-criteria distribution model for global software development projects', J. Brazilian Comput. Soc., vol. 16, no. 2, pp. 97-115, 2010.

[s24] T. Leonard, V. Berzins, and M. J. Holden, 'Gathering Requirements from Remote Users', pp. 462 471.

[s25] A. Hoffmann and C. Lescher, 'Collaboration and Intercultural Issues on Requirements: Communication, Understanding and Softskills (CIRCUS)', 2009 Collab. Intercult. Issues Requir. Commun. Underst. Softskills, CIRCUS 2009, no. Circus 2009, pp. 1-4, 2009.

[s26] G. N. Aranda, A. Vizcaíno, A. Cechich, and M. Piattini, 'Strategies to Minimize Problems in Global Requirements Elicitation RE-GSD Methodology', CLEI Electron. J., vol. 11, no. 1, 2008.

[s27] Darja Šmite, 'Requirements Management in Distributed Projects', Knowl. Creat. Diffus. Util., vol. 1, no. 2, pp. 69-76, 2006

[s28] J. Hanisch and B. Corbitt, 'Impediments to requirements engineering during global software development', Eur. J. Inf. Syst., vol. 16, no. 6, pp. 793 $805,2007$.

[s29] R. Lai and N. Ali, 'A Requirements Management Method for Global Software Development', Humanpub.Org, vol. 1, no. March, pp. 38-58, 2013.

[s30] R. E. Gallardo-valencia and S. E. Sim, 'Continuous and Collaborative Validation: A Field Study of Requirements Knowledge in Agile', 2010. 